\title{
Macroinvertebrados aquáticos associados às raízes de Eichhornia azuera (Swarts) Kunth (Pontederiaceae) em uma lagoa marginal no Pantanal, MS
}

\author{
Hugo Henrique L. Saulino ${ }^{1 *}$ \\ Susana Trivinho-Strixino ${ }^{2}$ \\ Universidade Federal de São Carlos \\ Rodovia Washington Luís (SP-310), km 235, CEP 13565-905, São Carlos - SP, Brasil \\ ${ }^{1}$ Programa de Pós-Graduação em Ecologia e Recursos Naturais \\ ${ }^{2}$ Laboratório de Ecologia de Insetos Aquáticos, Departamento de Hidrobiologia \\ * Autor para correspondência \\ hugosaulino@gmail.com
}

Submetido em 31/07/2014

Aceito para publicação em 08/04/2014

\section{Resumo}

Macrófitas aquáticas desempenham um importante papel na estruturação de comunidades dos ecossistemas de aquáticos. No presente estudo, a abundância e a riqueza da comunidade de macroinvertebrados foram comparadas através de correlação simples e da participação relativa média com o volume de raízes de Eichhornia azurea numa lagoa marginal no Pantanal (MS) no período de seca. Os macroinvertebrados foram identificados até o menor nível taxonômico possível e os volumes das raízes obtidos pelo método de deslocamento de volume. Foram identificados 21 táxons pertencentes a 13 famílias num total de 371 exemplares. Chironomus sp. (Chironomidae), Slavina sp. e Dero sp. (Naididae), todos detritívoros, foram mais representativos. Houve correlação positiva entre o volume das raízes com a abundância e riqueza de macroinvertebrados. Embora a análise de variância não tenha mostrado diferenças significativas entre riqueza e abundância da macrofauna e o volume das raízes, observou-se uma tendência de aumento de ambas as variáveis com o aumento do volume das raízes. Assim, as raízes maiores devem ter maior disponibilidade de microhabitats justificando a tendência observada. Os resultados obtidos indicaram que as dimensões das raízes $E$. azurea podem desempenhar importante papel ecológico como abrigo e local de acúmulo de alimentos para os macroinvertebrados em lagoas.

Palavras-chave: Fitofauna; Lagoa; Nicho; Zona litoral

\section{Abstract}

Aquatic macroinvertebrates associated with roots of Eichhornia azuera (Swarts) Kunth (Pontederiaceae) in an oxbow lake in Pantanal, MS. Macrophytes play an important role in the community structure of aquatic systems. In this study, we examined the macroinvertebrate communities within 20 Eichhornia azurea roots in an oxbow lake in Pantanal (MS) during dry season. Species abundance and richness were compared via linear regression, and the mean relative proportion of macroinvertebrates among root samples was determined. Macroinvertebrates were identified to the lowest practical taxonomic level, and root volume was measured using the volume displacement method. We identified 371 specimens, belonging to 31 families and 21 taxonomic groups. Chironomus sp. (Chironomidae), Slavinia sp. and Dero sp. (Naididae), all detritivores, 
were most the represented taxa. Regression analysis indicated a positive correlation between root volume and the abundance and richness of the macroinvertebrate community. Analysis of variance showed no statistically significant differences between species abundance and richness among different $E$. azuera root volumes, however we did observe a trend toward a positive correlation of both variables with increasing root volume. We suspect that greater root volumes should increase microhabitat availability, which could explain this observed trend. Our results indicate that E. azurea roots may play an important ecologic role (e.g., by providing shelter, food stock) in lake macroinvertebrate communities.

Key words: Littoral zone; Niche; Phytofauna; Pond

\section{Introdução}

A planície de inundação do Pantanal é uma dos maiores áreas alagáveis do mundo, onde, em decorrência da descontinuidade hídrica, são formadas diversas lagoas que durante o período de seca podem ficar isoladas (TANIGUCHI et al., 2004). As regiões litorâneas desses sistemas podem apresentar uma abundante e rica biodiversidade, podendo ser composta por diferentes espécies de macrófitas que encontram condições favoráveis para o seu desenvolvimento (THOMAZ, et al., 2009; ARAUJO et al., 2010). Este tipo de ambiente, em geral formado por numerosas espécies vegetais constitui um dos mais complexos compartimentos dos sistemas aquáticos dulciaquícolas e abrigam numerosa e diversa fauna, como macroinvertebrados e peixes (RESENDE, 2000; OBOLEWSKI, 2011). Estas macrófitas aquáticas desempenham importante papel na estruturação de ecossistemas de lagoas, contribuindo principalmente para o aumento da heterogeneidade ambiental (AGOSTINHO et al., 2003) e influenciando diretamente na estrutura de outras comunidades, levando, assim, ao aumento da biodiversidade local (THOMAZ et al., 2004; CREMONA et al., 2008).

Os macroinvertebrados aquáticos compõem uma das comunidades mais abundantes e diversificadas nas regiões litorâneas dos ecossistemas lênticos (KOUAMÉ et al., 2011). Sua distribuição varia conforme as características ambientais como, por exemplo, o tamanho da área, características químicas da água, tipo de sedimento, regimes de inundação e presença e de macrófitas aquáticas (HEINO, 2000; OBOLEWSKI, 2011). Macrófitas nas regiões litorâneas desempenham importante papel ecológico na estruturação da comunidade de macroinvertebrados associados (WALKER et al., 2013), proporcionando, além de local para deposição de ovos e refúgio contra predadores, fonte indireta de alimento para raspadores, que utilizam o biofilme que se adere às paredes do vegetal, e direta para as espécies minadoras e fragmentadoras (VILLANUEVA; TROCHINE, 2005), que utilizam o tecido vegetal como fonte de alimento (NEWMAN, 1991). A complexidade da estrutura morfológica e alta produção de biomassa desses vegetais são fatores que influenciam na estruturação da comunidade de macroinvertebrados aquáticos, alterando a abundância e a riqueza faunística através de maior ou menor disponibilidade de habitats (DIBBLE; THOMAZ, 2006) e também pela alteração das características abióticas da água, como por exemplo, pH e oxigênio (SILVA; HENRY, 2013). Eichhornia azurea é uma macrófita aquática enraizada com folhas flutuantes, que apresenta ampla distribuição nos ecossistemas de água doce das Américas e é popularmente conhecida como aguapé ou camalote (POTT; POTT, 2000). Suas raízes inseridas no caule se assemelham às longas cabeleiras que são compostas por diversas e finas ramificações. O comprimento de suas raízes pode apresentar grande variação, podendo apresentar poucos centímetros (5 $\mathrm{cm})$, no início de seu desenvolvimento, até atingir um metro, em plantas mais velhas (PADIAL et al., 2009). Esta espécie de macrófita geralmente favorece a presença de rica e abundante macrofauna, em vista de suas folhas servirem como fonte de alimento para espécies fragmentadoras quando em fase de senescência e, para as espécies coletoras, que obtém o recurso alimentar através do acumulo de detritos em suas raízes (TRIVINHOSTRIXINO et al., 2007; MARTINS et al., 2011).

Em vista de se obter mais conhecimento sobre a influência da macrófita de E. azurea sobre a comunidade de macroinvertebrados nos sistemas aquáticos continentais, o presente estudo teve como objetivo 
avaliar se a riqueza e abundância da comunidade de macroinvertebrados associada às raízes em um banco aguapé de uma lagoa marginal na região do Pantanal (MS) responde ao aumento no volume das raízes.

\section{Materiais e Métodos}

\section{Área de estudo}

O estudo foi realizado na baía da Medalha (19³4'33”S, 5700'51'W), situada na Base de Estudos do Pantanal (BEP), da Universidade Federal do Mato Grosso do Sul (Corumbá, MS). Esta é uma baía perene, com substrato tipo arenoso, possuindo conexão temporária com o rio Miranda por meio de uma vazante durante o período da cheia. A baía apresenta uma área total de $54.117 \mathrm{~m}^{2}$, com aproximadamente 25\% (13.469 $\mathrm{m}^{2}$ ) ocupada pela macrófita aquática Eichhornia azurea.

Em uma coleta no banco de E. azurea próximo ao litoral, foram amostradas aleatoriamente 20 raízes, no período de estiagem (setembro de 2010). As raízes foram cortadas cuidadosamente na inserção dos caules com utilização de uma tesoura de poda, logo após o desbaste da parte área da planta (Figura 1). Cada unidade (raiz) foi acondicionada em sacos plásticos com água do local. No laboratório, as raízes foram lavadas sob jatos d'água e o material retido em peneira com malha de $0,25 \mathrm{~mm}$. A fauna associada, triada em bandeja transiluminada, foi preservada em etanol $70 \%$, exceto Oligochaeta, previamente fixados em solução de formol 4\%. As identificações foram feitas até o menor nível taxonômico possível com o auxílio de chaves de identificação: Brinkurst e Marchese (1989), Nieser e Melo (1997), Costa et al. (2000), Domínguez et al. (2006), Lecci e Fröehlich (2007), Domínguez e Fernandez (2009) e Trivinho-Strixino (2011). Os diferentes táxons foram agrupados segundo as categorias funcionais de predadores, coletores, raspadores e fragmentadores (MERRITT; CUMMINS, 1996). Os volumes das raízes foram estimados em laboratório pelo método de deslocamento de volume de água. Numa proveta graduada (escala de $1 \mathrm{~mL}$ ), contendo água com volume inicial de $10 \mathrm{~mL}$, foram mergulhadas as raízes e registrados os deslocamentos de volume. As raízes foram agrupadas em cinco classes de volume $\left(\mathrm{cm}^{3}\right)$ : $>1<3, \geq 3<6, \geq 6<9, \geq 9<11$ e $\geq 11<20$.

FIGURA 1: Aspecto do caule e raízes de E. azurea com indicação de uma unidade amostral. Imagem adaptada de Padial et al. (2009).

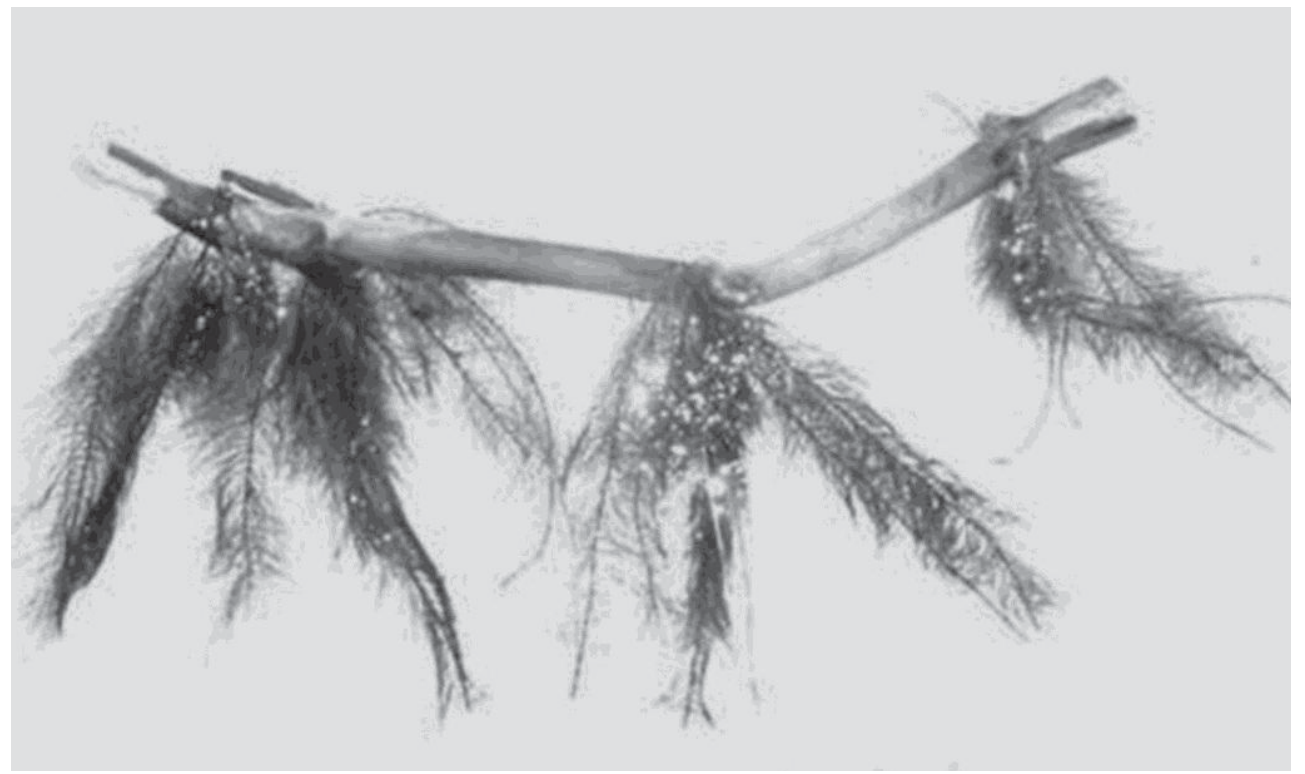




\section{Análise dos dados}

A comunidade associada às raízes de $E$. azurea foi analisada através da abundância, riqueza e participação relativa dos táxons e dos grupos funcionais em cada classe de volume de raíz. Os valores médios da abundância e da riqueza faunística foram comparados entre as diferentes classes de volumes de raízes através de uma linha de tendência linear, para prever se há uma tendência do aumento dessas variáveis com o aumento do volume. Os dados de abundância e riqueza total foram submetidos a uma análise de regressão linear simples com os volumes das raízes (GOTELLI; ELLISON, 2011), a fim de verificar a veracidade da hipótese. Os mesmos dados foram também submetidos à análise de variância (ANOVA), utilizando softwear PAST (Paleontological Statistc Softwear - versão 2.17) (HAMMER et al, 2001), a fim de se verificar se houve diferenças significativas na abundância e riqueza de macroinvertebrados aquáticos entre as diferentes classes de volumes de raízes de $E$. azurea.

\section{Resultados}

Foram identificados 371 espécimes em 21 táxons, pertencentes a 13 famílias. Os insetos representaram mais de $86 \%$ do total de indivíduos, a maioria com baixa participação numérica. Chironomus sp. (Chironomidae - Diptera) ( $\mathrm{n}=120,32 \%)$ e Derovatelus (Dysticidae - Coleoptera, $n=50,13 \%$ ) foram os táxons mais abundantes. Outros, como os oligoquetos Slavina sp. $(\mathrm{n}=51,13 \%)$ e Dero sp. $(\mathrm{n}=36,9 \%)$ (Naididae) também apresentaram valores numéricos mais elevados (Tabela 1). Os demais táxons apresentaram frequências muito baixas, com valores menores que $1 \%$ do total coletado. Entre as guildas alimentares houve maior participação relativa da categoria do grupo funcional dos coletores (69\%), seguido pelos predadores $(25 \%)$; os raspadores representaram apenas 5\% da fauna associada (Figura 2).

A análise dos valores médios de abundância e riqueza com as diferentes classes de raízes mostrou uma tendência do aumento destas variáveis com o aumento do volume de raíz (Figura 3). As regressões lineares

TABELA 1: Participação relativa dos táxons associados às raízes de E. azurea na baía da Medalha, Pantanal (MS).

\begin{tabular}{lcccccc}
\hline \multicolumn{1}{c}{ Taxons } & Gr. Func. & $>\mathbf{1}<\mathbf{3}$ & $\geq \mathbf{3}<\mathbf{6}$ & $\geq \mathbf{6}<\mathbf{9}$ & $\geq \mathbf{9}<\mathbf{1 1}$ & $\geq \mathbf{1 1 < 2 0}$ \\
\hline Dero sp. & Co & 1,67 & 10,24 & 2,86 & 2,60 & 4,49 \\
Slavina sp. & Co & 10,00 & 4,88 & 17,86 & 1,56 & 1,92 \\
Prístina sp. & Co & 0,83 & 0,24 & & & 1,28 \\
Caenis sp. & Co & 0,00 & 0,98 & 3,21 & 3,65 & 4,49 \\
Chironomus sp. & Co & 0,83 & 5,37 & 19,29 & 39,06 & 19,87 \\
Polypedilum sp. & Co & & 0,24 & 0,18 & & \\
Beardius sp. & Frag & & 0,98 & 0,18 & 2,08 & \\
Larsia sp. & Pre & 1,67 & 1,46 & 0,18 & & \\
Derovatelus sp. & Pre & 6,67 & 5,85 & 2,50 & 9,38 & 24,36 \\
Hetaerina sp. & Pre & 3,33 & 0,24 & & 1,56 & \\
Glossiphoniidae & Pre & 0,83 & & & & 0,64 \\
Octhterus sp. & Pre & & & 0,18 & 0,26 & \\
Belostoma sp. & Pre & & & & 0,26 & 0,64 \\
Libellula sp. & Pre & & & & 0,26 & 0,64 \\
Culex sp. & Pre & 5,00 & 0,73 & & & \\
Curicta sp. & Pre & & & 0,18 & & \\
Neoplea sp. & Pre & 4,17 & 4,88 & 0,18 & & \\
Heterelmis sp. & Ras & 0,83 & & 0,18 & 1,04 & 0,64 \\
Biomphalaria sp. & Ras & & & & 0,26 & 0,64 \\
Pomacea sp. & Ras & 1,67 & 0,24 & 0,18 & & 1,92 \\
\hline
\end{tabular}

Gr. Func. - Grupos funcionais, Co - coletor, Frag - fragmentador, Pre - predador, Ras - raspador. 
FIGURA 2: Abundância média dos grupos funcionais da macrofauna associada às raízes de E. azurea na baía da Medallha, Pantanal (MS).

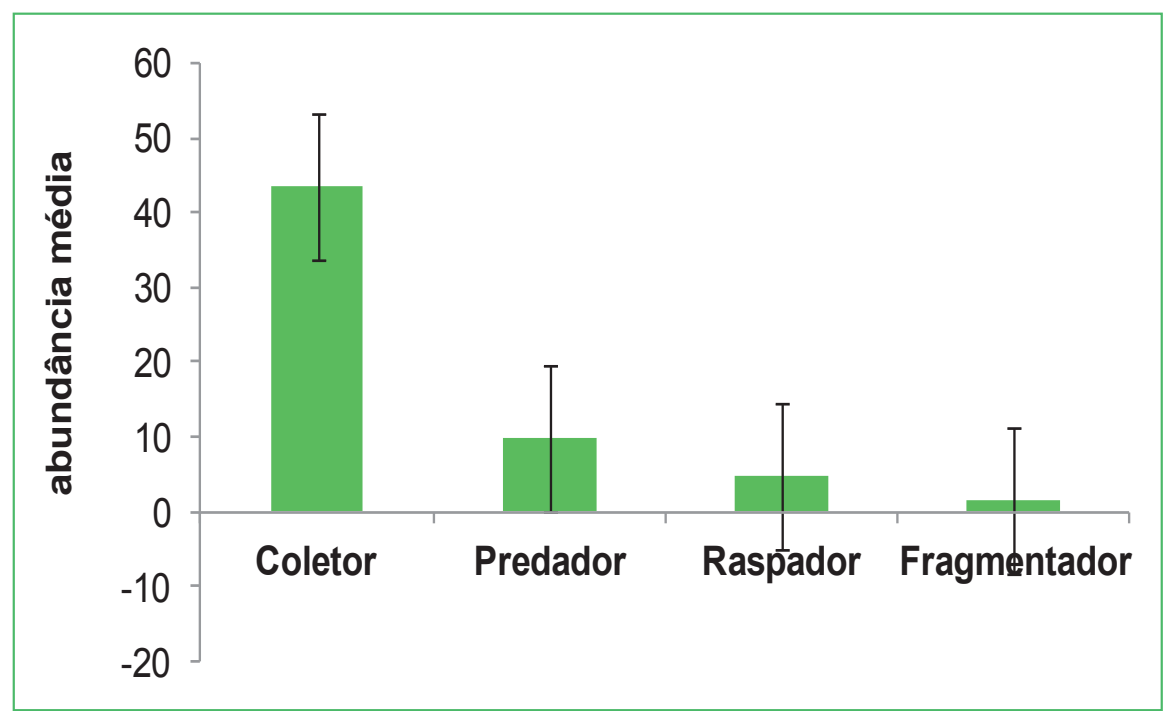

FIGURA 3: Abundância (A) e riqueza (B) médias dos táxons associados às diferentes classes de volumes de raízes de E. azurea na baía da Medalha - Pantanal ( MS).

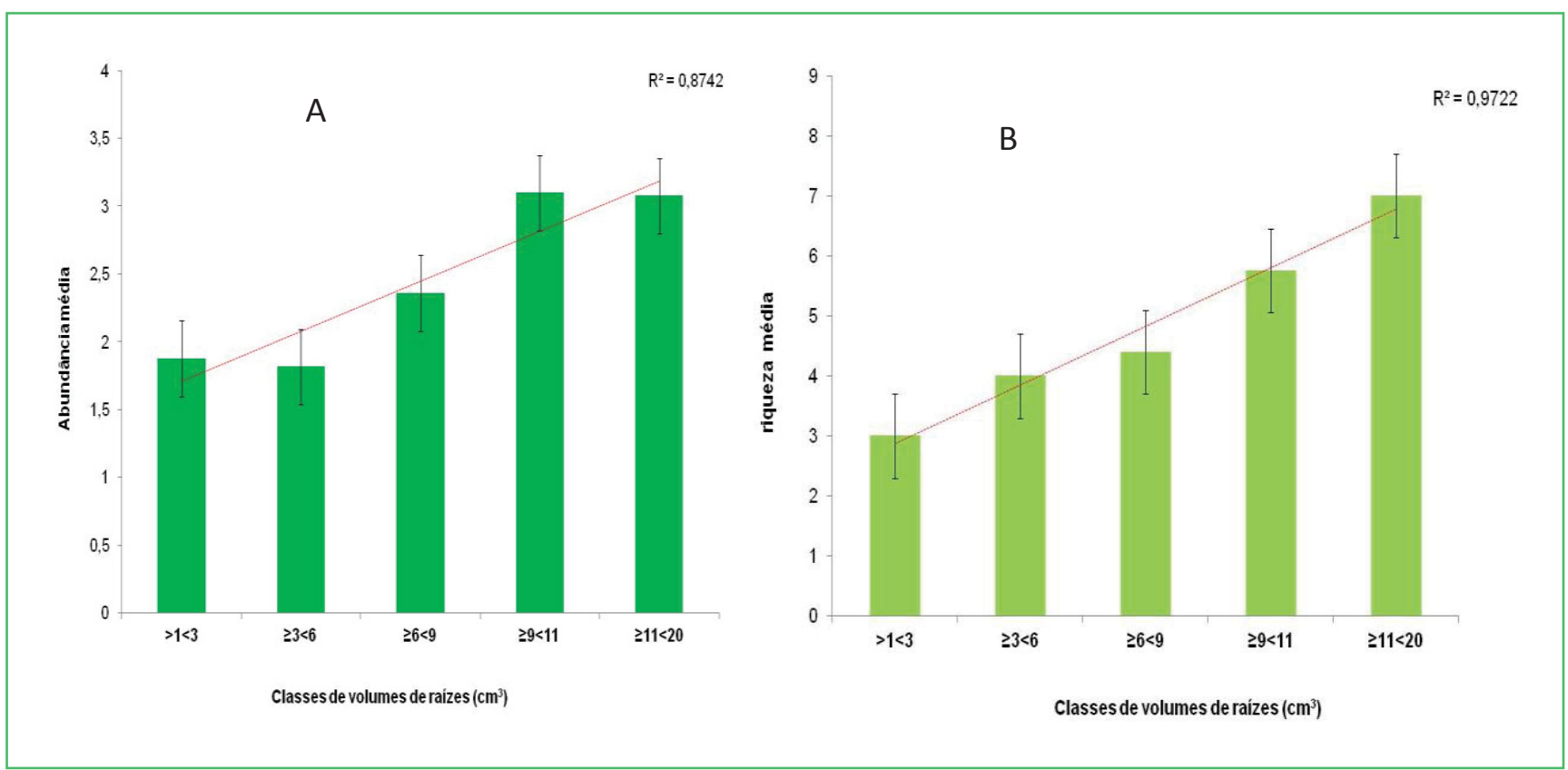

com as variáveis de abundância $\left(\mathrm{r}^{2}=0,30, \mathrm{p}=0,002\right)$ e indicou diferenças significativas na participação relativa riqueza faunísticas com o volume das raízes $\left(\mathrm{r}^{2}=0,15\right.$, e riqueza média de táxons entre as diferentes classes de $\mathrm{p}=0,002)$ (Figura 4) foram positivas, sendo porém, esta volume de raízes $(\mathrm{p}=0,9206, \mathrm{~F}=0,2305)$.

última uma correlação fraca. A análise de variância não 
FIGURA 4: Regressão linear entre abundância (A) e riqueza (B) de macroinvertebrados associados e volume de raiz de E. azurea, da baía da Medalha, Pantanal (MS).

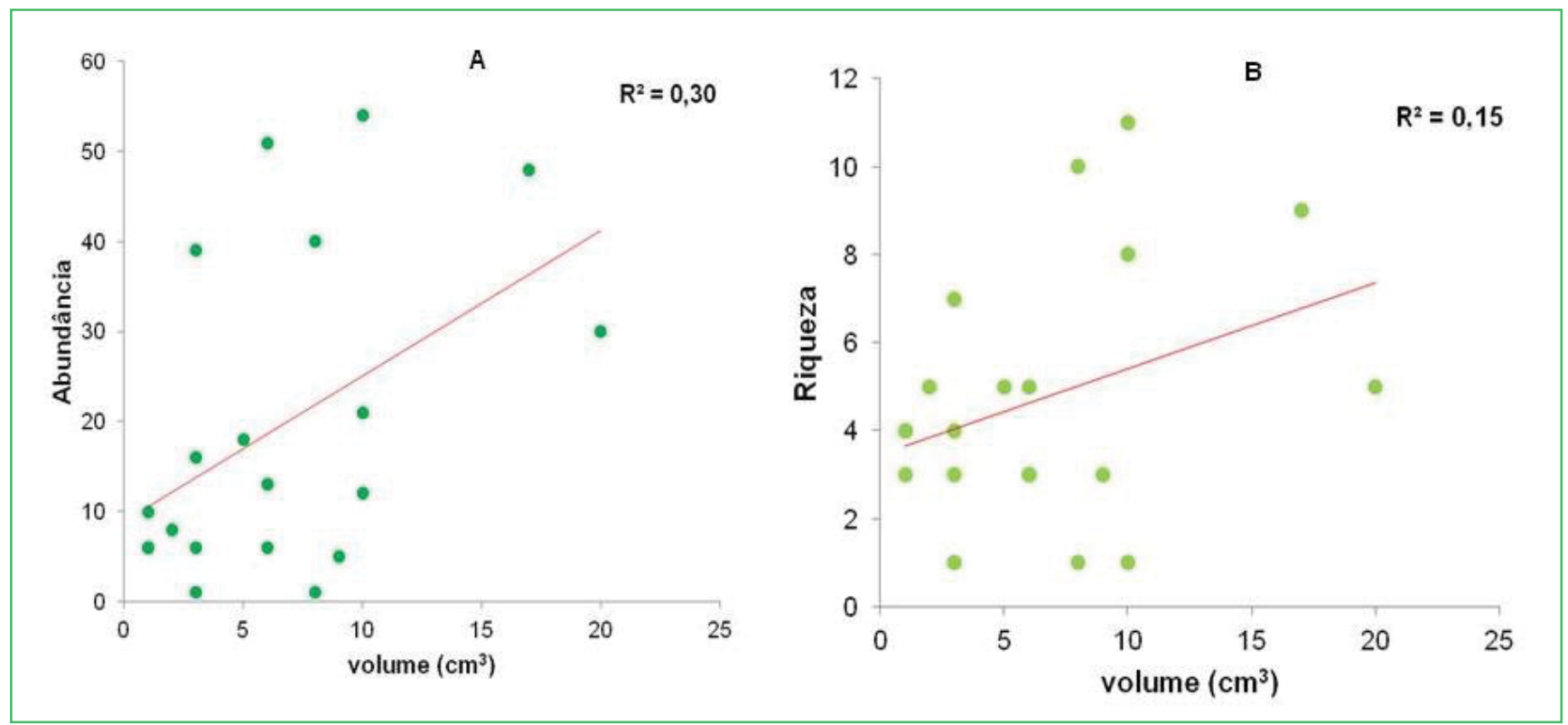

\section{Discussão}

O aumento da abundância e riqueza de macroinvertebrados com o aumento de volume de raiz de E. azurea se explica pelo maior disponibilidade de microhabitats resultantes do emaranhado de suas ramificações (TOWNSEND et al., 2010), que possibilita condições para o estabelecimento de uma rica e abundante comunidade (SCHEFFER, 2004; THOMAZ; CUNHA, 2010). As raízes de E. azurea, embora muito ramificadas, apresentam espaços muito pequenos, que favorecem a colonização por macroinvertebrados de menor porte. Este fato explica a dominância de táxons das famílias Chironomidae (Chironomus sp.) e Naididae (Dero sp. e Slavina sp.) que contribuíram para a regressão positiva obtida neste estudo. Estes táxons costumam apresentar elevada riqueza nos ecossistemas de água doce e, em particular, na fauna fitófila (LEARNER et al., 1978; PINDER, 1986; RODRIGUES et al., 2013), sendo também dominantes em bancos de macrófitas aquáticas (PEARSON; MATTHEWS, 1995; TRIVINHO-STRIXINO et al., 2000; KOUAMÉ et al., 2011; MARTINS et al., 2011) onde encontram boa disponibilidade de recursos alimentares na forma de detritos orgânicos aderidos às raízes. Outras variáveis ambientais da lagoa, como, por exemplo, maior adensamento das macrófitas, proximidade das raízes do vegetal com áreas de águas abertas ou fechadas e modificações da profundidade da lagoa, poderiam também ter influenciado na distribuição desta macrofauna entre as raízes, contribuindo para diferentes distribuições de riqueza (SYCHRA et al., 2010).

A presença de determinados grupos funcionais nas raízes, como por exemplo, predadores, poderia ser também outro fator determinante na riqueza da comunidade associada. Sua elevada participação teve relevância na estruturação da comunidade fitófila analisada. Em decorrência da busca por suas presas, a presença desse grupo funcional pode influenciar na ocupação por espaço (SIH, 1982) e, assim, na redução de outros táxons. Pode-se inferir no presente estudo que, a guilda dos predadores, foi uma variável que influenciou na riqueza da comunidade associada às raízes.

O maior volume das raízes $E$. azurea na baía da Medalha (MS) deve proporcionar maior disponibilidade de microhabitats para a fauna de macroinvertebrados levando, assim, ao aumento da abundância e riqueza faunística. Além disso, presença de grupos predadores 
pode ser um importante fator na estruturação da comunidade macroinvertebrados associados às suas raízes.

\section{Agradecimentos}

À Universidade Federal do Mato Grosso do Sul e toda equipe de coordenação do XIII Curso de Campo Ecologia do Pantanal na realização deste trabalho.

\section{Referências}

AGOSTINHO, A. A.; GOMES, L. C.; JULIO JR., H. F. Relações entre macrófitas e fauna de peixes. In: THOMAZ, S. M.; BINI, L. M. (Ed.). Ecologia e manejo de macrofitas aquáticas. Maringá: EDUEM. 2003. p. 261-279.

ARAUJO, R. B.; LANGEANI, F.; RANGA, N. T. Vascular plants of oxbow lakes of Turvo River, Upper Paraná River Basin, São Paulo State, Brazil. Check List: Journal of Species and Distribution, Rio Claro, v. 6, n. 1, p. 58-61, 2010.

BRINKHURST, R. O.; MARCHESE, M. R. Guia para la indentificacion de Oligoquetos aquáticos continentales de Sud y Centroamérica. Santa Fé: Climax, 1989. 207 p.

COSTA, J. M.; MACHADO, A. B. M.; LENCIONI, F. A. A.; SANTOS, T. C. Diversidade e distribuição dos Odonata (Insecta) no estado de São Paulo, Brasil: Parte I - Lista das espécies e registros bibliográficos. Publicações Avulsas do Museu Nacional do Rio de Janeiro, Rio de Janeiro, v. 80, p. 1-27, 2000.

CREMONA, F.; PlANAS, D.; LUCOTTE, M. Biomass and composition of macroinvertebrate communities associated with different types of macrophyte architectures and habitats in a large fluvial lake. Fundamental and Applied Limnology, Stuttgart, v. 2, n. 171, p. 119-130, 2008.

DIBBLE, E. D.; THOMAZ, S. M. A simple method to estimate spatial complexity in aquatic plants. Brazilian Archivies of Biology and Technology, Curitiba, v. 49, n. 3, p. 421-428, 2006.

DOMÍNGUEZ, E.; FERNANDEZ, H. R. Macroinvertebrados bentónicos sudamericanos: sistemática y biología. Tucuman: Fundación Miguel Lillo, 2009. p. 411-468.

DOMÍNGUEZ, E.; MOLINERI, C.; PESCADOR, M. L.; HUBBARD, M. D.; NIETO, C. Aquatic Biodiversity in Latin America: Ephemeroptera of South America. Sofia: Ed. Pensoft. 2006. $646 \mathrm{p}$.

GOTELli, N. J.; ELlisON, A. M. Princípios de estatística em ecologia. Porto Alegre: ARTMED, 2011. 527 pp.

HAMMER, Ø.; HARPER, D. A. T.; RYAN, P. D. PAST: Paleontological statistics software package for education and data analysis. 2001. Disponível em: <http://palaeo-electronica. org/2001_1/past/issue1_01.htm>. Acesso em: 2 abr. 2014.

HEINO, J. Lentic macroinvertebrates assemblages structure along gradients in special heterogeneity, habitat size and water chemistry. Hydrobiologia, Brussels, v. 418, p. 229-242, 2000.
KOUAMÉ, M. K.; DIETOA, M. Y.; EDIA, E. O.; DA COSTA, S. K.; OUATTARA, A.; GOURÈNE, G. Macroinvertebrate communities associated with macrophyte habitats in a tropical man-made lake (Lake Taabo, Côte d'Ivoire). Knowledge and Management of Aquatic Ecosystems, Paris, v. 3, n. 400, p. 3-18, 2011.

LEARNER, M. A.; LOCHHEAD, G.; HUGHES, B. D. A review of the biology of British Naididae (Oligochaeta) with emphasis on the lotic environment. Freshwater Biology, London, v. 8, p. 357$375,1978$.

LECCI, L. S.; FRÖEHLICH, C. G. Plecoptera. In: FROËHLICH, C. G. (Org.). Guia on-line: identificação de larvas de insetos aquáticos do estado de São Paulo. 2007. Disponível em: <http:// sites.ffclrp.usp.br/aguadoce/guiaonline>. Acesso em: 10 dez. 2010. MARTINS, R. T.; SILVEIRA, L. S.; ALVES, R. G. Colonization by oligochaetes (Annelida: Citellata) in decomposing leaves of Eichhornia azurea (SW.) Kunth (Pontederiaceae) in a tropical lentic system. Annales de Limnologie - International Journal of Lymnologi, Santa Fé, v. 47, p. 339-346, 2011.

MERRITT, R. W.; CUMMINS, K. W. An introduction to the aquatic insects of North America. Dubuque: Kendall/Hunt Company, 1996. $862 \mathrm{p}$.

NEWMAN, R. N. Herbivory and detritivory on freshwater macrophytes by invertebrates: a review. Journal of the North American Benthological Society, Lawrence, v. 2, n. 10, p. 89-114, 1991.

NIESER, N.; MELO, A. L. Os heterópteros aquáticos de Minas Gerais: guia introdutório com chave de indentificação para as espécies de Neomorpha; Gerromorpha. Belo Horizonte: UFMG, 1997. $180 \mathrm{p}$.

OBOLEWSKI, K. Macroinvertebrates patterns along environmental gradients and hydrological connectivity of oxbow lakes. Ecological Engineering, New York, v. 37, p. 796-805, 2011.

PADIAL, A. A.; THOMAZ, S. M.; AGOSTINHO, A. A. Effects of structural heterogeneity provided by the floating macrophyte Eichhornia azurea on the predation efficiency and habitat use of small Neotropical fish Moenkhausia sanctaefilomenae. Hydrobilogia, Brussels, v. 624, p. 161-170, 2009.

PARSONS, J. K.; MATTHEWS, R. A. Analysis of the associations between macroinvertebrates and macrophytes in a freshwater pond. Northwest Science, Washington, v. 69, n. 4, p. 265-275, 1995.

PINDER, L. C. V. Biology of freshwater Chironomidae. Annual Review of Entomology, Palo Alto, v. 31, p. 1-23, 1986.

POTT, V. J.; POTT, A. Plantas aquáticas do Pantanal. Brasília: Embrapa - Centro de Pesquisa Agropecuária do Pantanal, 2000. $404 \mathrm{p}$.

RESENDE, E. K. Trophic structure of fish assemblages in the lower Miranda river, Pantanal, Mato Grosso do Sul State, Brazil. Brazilian Journal of Biology, São Carlos, v. 60, n. 3, 389-403, 2000.

RODRIGUES, L. F. T.; LEITE, F. S.; ALVES, R. G. Inventory and distribution of Oligochaeta (Annelida, Citellata) in first order streams in preserved areas of the state of Minas Gerais, Brazil. Biota Neotropica, Campinas, v. 13, n. 1, p. 245-254, 2013.

SCHEFFER, M. Ecology of shallow lakes. Norwell: Kluwer Academic Publishers, 2004. 357 p. 
SIH, A. Foraging Strategies and the avoidance of predation by an aquatic insect, Notonecta hoffmanni. Ecology, New York, v. 63, n. 3, p. 786-796, 1982.

SILVA, C. V.; HENRY, R. Aquatic macroinvertebrates associated with Eichhornia azurea (Swatrs) Khunt and relationships with abiotic factors in marginal lentic ecosystems (São Paulo, Brazil). Brazilian Journal of Biology, São Carlos, v. 73, n. 1, p. 149-162, 2013.

SYCHRA, J.; ADAMÉK, Z.; PETŘIVALSKÁ. Distribuiton and diversity of littoral macroinvertebrates within extensive reed beds of lowlandpond. Annales de Limnologie - International Journal of Lymnologi, Santa Fé, v. 46, p. 281-289, 2010.

TANIGUCHI, G. M.; BICUDO, D. C.; SENNAB, P. A. C. Abiotic variables in Littoral-Limnect gradient of an Oxbow Lake of Mogi-Guaçú River Foodplain, Southeastern, Brazil. Brazilian Archivies of Biology and Technology, Curitiba, v. 47, n. 6, p. 961-971, 2004.

THOMAZ, S. M.; CARVALHO, P.; PADIAL, A. A.; KOBAYASHI, J. T. Temporal and spatial patterns of aquatic macrophyte diversity in the Upper Paraná River floodplain. Brazilian Journal of Biology, São Carlos, v. 69, n. 2, p. 617-625, 2009.

THOMAZ, S. M.; CUNHA, E. R. D. The role of macrophytes in habitat structuring in aquatic ecosystems: methods of measurement, causes and consequences on animal assembleges's composition and biodiversity. Acta Limnologica Brasiliensia, São Carlos, v. 22, n. 2, p. 218-236, 2010.

THOMAZ, S. M.; PAGIORO, T. A.; BINI, L. M.; SOUZA, D. C. Aquatic macrophytes of the Upper Paraná River foodplain: species list and patterns of diversity in larges scales. In: AGOSTINHO, A.; RODRIGUES, L.; GOMES, L. C.; THOMAZ, S. M.; MIRANDA, L. E. (Org.). Strucutre and functioning of the Paranpa River and its foodplain. Maringá: EDUEM. 2004. p. 221-225.
TOWNSEND, C. R.; BEGON, M.; HARPER, J. L. Fundamentos em ecologia. Porto Alegre: Artmed, 2010. 576 p.

TRIVINHO-STRIXINO, S. Larvas de Chironomidae: guia de identificação. São Carlos: Departamento de Hidrobiologia/Lab. De Entomologia Aquática, Universidade Federal de São Carlos, 2011. $377 \mathrm{p}$.

TRIVINHO-STRIXINO, S.; CORREIA, L. C. S.; SONODA, K. Phytophilous Chironomidae (Diptera) and other macroinvertebrates in the ox-bow Infernão lake (Jataí Ecological Station, Luiz Antônio, SP, Brazil). Brazilian Journal of Biology, São Carlos, v. 60, n. 3, p. 527-535, 2000.

TRIVINHO-STRIXINO， S.; RODRIGUES-PELAEZ, M.; JANCSO, M. A. Chironomidae associados a Eichhornia azurea (Swarts) Khunt: influência da profundidade do corpo d'água na estrutura da comunidade fitófila de lagoas marginais do Rio Mogi-Guaçu (Luiz Antonio, SP). In: SIMPÓSIO DE ECOLOGIA do PPGERN/UFSCar, I, 2007, São Carlos. Anais Cadernos de Resumos expandidos, São Carlos: PPGERN/UFSCar, 2007. p. 2633 ,

VILLANUEVA, V. D.; TROCHINE, C. The role of microorganisms in the diet of verger CF. Limnophilus (Trichoptera: Limnephilidae) larvae in the Patagonian Andean temporary pond. Wetlands, Madson, v. 25, n. 2, p. 473-479, 2005.

WALKER, P. D.; WIJNHOVEN, S.; VELDE, G. v.d. Macrophyte presence and growth form influence macroinvertebrate community strucuture. Aquatic Botany, New York, v. 104, p. 80-87, 2013. 